\title{
Ramucirumab as a second-line treatment for hepatocellular carcinoma: reaching out further to patients with elevated alpha-fetoprotein
}

\author{
Edoardo G. Giannini ${ }^{1}$, Franco Trevisani ${ }^{2}$ \\ ${ }^{1}$ Gastroenterology Unit, Department of Internal Medicine, Ospedale Policlinico San Martino IRCCS per l'Oncologia, University of Genoa, Genoa, \\ Italy; ${ }^{2}$ Medical Semeiotics Unit, Sant'Orsola Hospital, Alma Mater Studiorum University of Bologna, Bologna, Italy \\ Correspondence to: Prof. Franco Trevisani. Department of Medical and Surgical Sciences, Semeiotica Medica, Alma Mater Studiorum, University of \\ Bologna, Bologna, Italy. Email: franco.trevisani@unibo.it. \\ Comment on: Zhu AX, Kang YK, Yen CJ, et al. Ramucirumab after sorafenib in patients with advanced hepatocellular carcinoma and increased \\ $\alpha$-fetoprotein concentrations (REACH-2): a randomised, double-blind, placebo-controlled, phase 3 trial. Lancet Oncol 2019;20:282-96.
}

Submitted Apr 01, 2019. Accepted for publication Apr 24, 2019.

doi: 10.21037/hbsn.2019.04.19

View this article at: http://dx.doi.org/10.21037/hbsn.2019.04.19

Hepatocellular carcinoma (HCC) occurs at a rate of 3-5\% per year in patients with compensated cirrhosis, and it often represents the first disease complication and the main cause of death in these patients (1). Surveillance for HCC is recommended by all liver disease associations, as it increases patient survival mainly as a result of early diagnosis of the tumour, allowing most patients to convey to curative treatments $(2,3)$. However, the actual uptake of surveillance for HCC in clinical practice is low, so that a large proportion of patients are diagnosed with HCC at an advanced disease stage $(4,5)$. Moreover, HCC often recurs even after curative treatments or progresses to more advanced stages after non-curative treatments, making the therapeutic decision for this cancer a complex, dynamic and step-by-step process (6). Lastly, biology of HCC is poorly understood and often unpredictable. Indeed, in some patients-even under surveillance-this tumour may present as a tiny nodule that has already invaded a portal vein branch, or as a multinodular, bi-lobar disease, or as a metastatic disease, thus preventing any curative treatment approach. Before 2008, the majority of patients with these dismal characteristics were deemed unsuitable for any therapy and managed with best supportive care. Following the availability of sorafenib-which proved to be able to significantly improve the survival of patients with compensated liver disease and advance stage HCCtheir management was significantly improved (7). Indeed, the median survival of these patients, if untreated, is
7 months on the average and it was increased by sorafenib by approximately 3 months, corresponding to a $31 \%$ improvement [hazard ratio (HR) 0.69; 95\% CI: 0.55-0.87] $(7,8)$. Unfortunately, at that time and until recently, no effective second-line treatment was available for patients intolerant to sorafenib or who progressed under this therapy, despite the fact that several studies assessed the efficacy of new molecules in these patients (9). In this regard, regorafenib was the first drug that proved to be able to further extend, by approximately 3 months as compared to placebo, the survival of patients tolerant to sorafenib but who progressed under this treatment $(10.6$ vs. 7.8 months; HR 0.63; 95\% CI: $0.50-0.76$; $\mathrm{P}<0.001)(10)$. Therefore, the sequence sorafenib-regorafenib extends to 26 months the median survival of patients with preserved liver function and not amenable to loco-regional treatments for HCC, compared to a life expectancy of 19 months when the second-line therapy is not used (11). Such a cumulative benefit cannot be disregarded, even from a clinical standpoint. However, although the sequence sorafenibregorafenib represents a cornerstone in the management of advanced HCC, it does not fulfil the need of patients intolerant to sorafenib (11). More recently, cabozantinib filled this therapeutic gap, leading to an improved survival of patients, compared to placebo, who either progressed or were intolerant to sorafenib (10.2 vs. 8.0 months; HR 0.76; 95\% CI: 0.63-0.92; $\mathrm{P}=0.005)(12)$.

Ramucirumab is a fully-human $\operatorname{IgG}_{1}$ monoclonal 
antibody selectively binding vascular endothelial growth factor receptor-2 (VEGFR-2) that, when used as firstline treatment in a phase II study, succeeded in providing a median progression free survival of 4.3 months and a disease control rate of $50 \%$ in HCC patients with preserved liver function (13). Following these encouraging results, a randomised, placebo-controlled, phase III study (REACH) was carried out (14). Unfortunately, this study, evaluating the efficacy of ramucirumab $(8 \mathrm{mg} / \mathrm{kg}$ given intravenously every 2 weeks) as second-line treatment in a large population of HCC patients with compensated cirrhosis (either progressors or intolerant to sorafenib) did not meet its primary end-point. In fact, the median overall survival was not significantly different between ramucirumab and placebo arms (9.2 vs. 7.6 months; HR 0.87; 95\% CI: $0.72-1.05 ; \mathrm{P}=0.14)$. Nevertheless, post-hoc analyses identified a pre-specified subgroup of patients, i.e., those with a baseline serum alpha-fetoprotein (AFP) $\geq 400 \mathrm{ng} / \mathrm{mL}$, who experienced a benefit from ramucirumab compared to placebo (median overall survival: 7.8 vs. 4.2 months; HR 0.67; 95\% CI: 0.51-0.90). The identification of this patient subgroup was clinically and biologically relevant for at least three reasons: firstly, patients with elevated AFP represent approximately $40 \%$ of those who show a tumor progression or do not tolerate sorafenib $(12,14)$. Secondly, elevated AFP levels identify tumours with a more aggressive clinical phenotype, characterised by multi-nodularity, larger lesions, vascular invasion, and poorer differentiation, while at the molecular level its expression seems to be associated with a peculiar intracellular oncogenic pathway activation $(15,16)$. Lastly, unlike inclusion of "all - comers", the selection of patients based on clinical biomarkers or hypothetical oncogenic drivers increases the likelihood that the therapeutic target is present, thereby enhancing the chance of a positive treatment outcome $(9,17)$.

Based on the potential benefit of ramucirumab in patients with high AFP levels, a second phase III randomized, placebo-controlled, study was planned (REACH-2) and carried out at 92 centres spread over 20 countries (18). It included 197 HCC patients who were intolerant (17\%) or experienced cancer progression $(83 \%)$ during sorafenib therapy and with AFP levels $\geq 400 \mathrm{ng} / \mathrm{mL}$. Sorafenib was the only previous systemic treatment allowed. About one third of patients had macrovascular invasion and $72 \%$ extrahepatic spread of the tumour. This study confirmed that the intravenous administration of $8 \mathrm{mg} / \mathrm{kg}$ of ramucirumab every 2 weeks significantly increases the median overall survival as compared to placebo (8.5 vs. 7.3 months; HR
0.71; 95\% CI: 0.53-0.95; $\mathrm{P}=0.0199)$, with an acceptable safety profile. Notably, these patients, despite a baseline well compensated cirrhosis (Child-Pugh A class) and a preserved clinical status (ECOG performance status $0-1$ ), showed an overall dismal prognosis, consistently with what observed in patients with similar AFP values enrolled in the REACH trial (14). Indeed, AFP, macrovascular invasion and ECOG performance status were the independent determinants of patient prognosis, and the regression analysis showed that AFP remained a strong negative prognostic factor even beyond the threshold of $400 \mathrm{ng} / \mathrm{mL}$. These results once more emphasize the ability of high AFP levels to herald an aggressive disease, even when patients are equalised by the other main prognostic factors.

In order to expand the sample size, the authors also pooled individual patient data from REACH and REACH-2 studies, obtaining a population of 542 patients (316 randomly assigned to ramucirumab and 226 to placebo). With this artifice, the ramucirumab efficacy improved (median overall survival: 8.1 vs. 5.0 months; HR 0.69; $95 \%$ CI: 0.57-0.84; $\mathrm{P}=0.0002$ ), mainly because of the poorer survival of the placebo arm compared to the REACH-2 study.

Despite these encouraging results provide a glimpse of hope for the second-line treatment of patients with a particularly aggressive disease, several questions remain open. First, also the efficacy of cabozantinib was relatively more pronounced in patients with an AFP $\geq 400 \mathrm{ng} / \mathrm{mL}$ (HR 0.71 ; 95\% CI: 0.54-0.94) than in those below this threshold (12). Hence, whether the results of the REACH-2 trial are a product of a biomarker-driven study or, instead, simply represent a general epiphenomenon of a still poorly characterised disease remains to be established. This conundrum is further heightened by the observation that some HCC subtypes with high epithelial cell adhesion molecule (epCAM) and AFP expression are characterised by elevated VEGF expression, suggesting that this particularly aggressive HCC subtypes may benefit from VEGF-targeted therapy (19). On the other hand, no definite correlation exists between expression of VEGF receptors (highly variable in HCC) and treatment outcome in HCC patients. Second, the exclusion of patients with "at-risk varices" from the REACH-2 study due to the risk of increasing haemorrhagic events-more frequently observed in the ramucirumab arm $(32.5 \%)$ than in the placebo arm (19.9\%) of the REACH trial-further limits the translation of its results to real-life clinical practice (14). Third, the improvement in median survival provided by 
ramucirumab, although statistically significant, should be interpreted in a broader context, considering its clinical relevance and cost-effectiveness. It is pertinent to note that, although the relative benefit over placebo (HR) obtained with ramucirumab is similar to those reported for regorafenib and cabozantinib as second-line therapy for HCC, the absolute median survival gain was much lower, being only 1.2 months. The authors attributed this limited improvement to a longer-than-expected survival of patients in the placebo group (7.3 months), likely due to their lower baseline AFP levels compared to the ramucirumab group, and results of post-hoc and pooled analyses give support to this hypothesis. Therefore, further work is needed to measure the impact of AFP levels on the result of systemic therapy for HCC, in an attempt to define the biomarker threshold beyond which survival gain becomes clinically futile. Lastly, the three cases of treatment-related kidney failure seen in the ramucirumab group represent an alert that should be taken into account when this therapy will enter into clinical practice.

In conclusion, the results of the REACH-2 study, identifying a peculiar subgroup of HCC patients who can benefit most from ramucirumab administration, further expands the current therapeutic armamentarium of clinicians facing the loss of response or intolerance to sorafenib. Namely, ramucirumab is a new pharmacological bullet against advanced HCC in patients with elevated AFP who, however, show an inherent poor prognosis even under this therapy.

\section{Acknowledgments}

None.

\section{Footnote}

Conflicts of Interest: The authors have no conflicts of interest to declare.

\section{References}

1. Sangiovanni A, Prati GM, Fasani P, et al. The natural history of compensated cirrhosis due to hepatitis $\mathrm{C}$ virus: A 17-year cohort study of 214 patients. Hepatology 2006;43:1303-10.

2. Italian Association for the Study of the Liver (AISF); AISF Expert Panel; AISF Coordinating Committee, et al. Position paper of the Italian Association for the Study of the Liver (AISF): the multidisciplinary clinical approach to hepatocellular carcinoma. Dig Liver Dis 2013;45:712-23.

3. Giannini EG, Cucchetti A, Erroi V, et al. Surveillance for early diagnosis of hepatocellular carcinoma: how best to do it? World J Gastroenterol 2013;19:8808-21.

4. Im S, Jang ES, Lee JH, et al. Surveillance rate and its impact on survival of hepatocellular carcinoma patients in South Korea: a cohort study. Cancer Res Treat 2019. [Epub ahead of print].

5. Simmons OL, Feng Y, Parikh ND, et al. Primary care provider practice patterns and barriers to hepatocellular carcinoma surveillance. Clin Gastroenterol Hepatol 2019;17:766-73.

6. Vitale A, Farinati F, Noaro G, et al. Restaging patients with hepatocellular carcinoma before additional treatment decisions: a multicenter cohort study. Hepatology 2018;68:1232-44.

7. Llovet JM, Ricci S, Mazzaferro V, et al. Sorafenib in advanced hepatocellular carcinoma. N Engl J Med 2008;359:378-90.

8. Giannini EG, Farinati F, Ciccarese F, et al. Prognosis of untreated hepatocellular carcinoma. Hepatology 2015;61:184-90.

9. Llovet JM, Hernandez-Gea V. Hepatocellular carcinoma: reasons for phase III failure and novel perspectives on trial design. Clin Cancer Res 2014;20:2072-9.

10. Bruix J, Qin S, Merle P, et al. Regorafenib for patients with hepatocellular carcinoma who progressed on sorafenib treatment (RESORCE): a randomised, double-blind, placebo-controlled, phase 3 trial. Lancet 2017;389:56-66.

11. Finn RS, Merle P, Granito A, et al. Outcomes of sequential treatment with sorafenib followed by regorafenib for HCC: Additional analyses from the phase III RESORCE trial. J Hepatol 2018;69:353-8.

12. Abou-Alfa GK, Meyer T, Cheng AL, et al. Cabozantinib in patients with advanced and progressing hepatocellular carcinoma. N Engl J Med 2018;379:54-63.

13. Zhu AX, Finn RS, Mulcahy M, et al. A phase II and biomarker study of ramucirumab, a human monoclonal antibody targeting the VEGF receptor-2, as first-line monotherapy in patients with advanced hepatocellular cancer. Clin Cancer Res 2013;19:6614-23.

14. Zhu AX, Park JO, Ryoo BY, et al. Ramucirumab vs. placebo as second-line treatment in patients with advanced hepatocellular carcinoma following first-line therapy with sorafenib (REACH): a randomised, double-blind, multicentre, phase 3 trial. Lancet Oncol 2015;16:859-70.

15. Giannini EG, Sammito G, Farinati F, et al. Determinants 
of alpha-fetoprotein levels in patients with hepatocellular carcinoma: implications for its clinical use. Cancer 2014;120:2150-7.

16. Hoshida Y, Nijman SM, Kobayashi M, et al. Integrative transcriptome analysis reveals common molecular subclasses of human hepatocellular carcinoma. Cancer Res 2009;69:7385-92.

17. Bitzer M, Horger M, Giannini EG, et al. Resminostat plus sorafenib as second-line therapy of advanced hepatocellular carcinoma - The SHELTER study. J Hepatol 2016;65:280-8.

Cite this article as: Giannini EG, Trevisani F. Ramucirumab as a second-line treatment for hepatocellular carcinoma: reaching out further to patients with elevated alpha-fetoprotein. HepatoBiliary Surg Nutr 2019;8(5):515-518. doi: 10.21037/ hbsn.2019.04.19
18. Zhu AX, Kang YK, Yen CJ, et al. Ramucirumab after sorafenib in patients with advanced hepatocellular carcinoma and increased $\alpha$-fetoprotein concentrations (REACH-2): a randomised, double-blind, placebocontrolled, phase 3 trial. Lancet Oncol 2019;20:282-96.

19. Shan YF, Huang YL, Xie YK, et al. Angiogenesis and clinicopathologic characteristics in different hepatocellular carcinoma subtypes defined by EpCAM and alpha-fetoprotein expression status. Med Oncol 2011;28:1012-16. 